\title{
Base-Station Random Placement Effect on the Accuracy of Ultrawideband Body-Centric Localization Applications
}

\author{
Richa Bharadwaj ${ }^{\circledR}$, Member, IEEE, Sritrija Swaisaenyakorn, Member, IEEE, \\ John C. Batchelor ${ }^{\circledR}$, Senior Member, IEEE, Shiban K. Koul ${ }^{\circledR}$, Fellow, IEEE, \\ and Akram Alomainy ${ }^{(D)}$, Senior Member, IEEE
}

\begin{abstract}
This letter presents an investigation on the effect of random placement of base stations on three-dimensional bodycentric localization in the ultrawideband frequency range. Different base station configurations are studied (varied in $x y z$-axis, $x y$-axis, and $z$-axis direction), keeping the same range estimation error values as obtained from the body-centric localization experimental data in order to keep the main focus on the influence of the base-station configurations. It is observed that random positions of the base stations in the $z$-axis lead to higher localization error $(3-8 \mathrm{~cm})$ as the target nodes are spread out more in the $z$-axis direction. The localization error is analyzed in terms of geometric, horizontal, vertical dilution of precision (DOP), which is a measure of effectiveness of the placement of the base stations. For the DOP values (5-15), 1-3 cm error reduction is observed, and for higher DOP $(>20)$ values, the error increases by 3-5 cm for shortrange communication. Further analysis is carried out by varying the range estimation error in terms of mean value and standard deviation for evaluating the random base-station configurations, which also plays an important role in the accuracy of estimating the location of the target.
\end{abstract}

Index Terms-Body-centric communication, dilution of precision (DOP), localization, ultrawideband (UWB), wearable antennas.

\section{INTRODUCTION}

$\mathbf{L}$ OCALIZATION and tracking using the compact antennas have attracted intensive research and development attention in recent years [1], [2]. With the miniaturization of devices and sophisticated communication systems suited for numerous applications, human motion tracking and localization are active areas of research benefiting consumers in day-to-day life [2]-[4]. Some applications include asset tracking, health care, patient monitoring, sports, industrial and transport, hazard warnings, real-time monitoring, search, and rescue [2], [5]-[7].

R. Bharadwaj and S. K. Koul are with the Microwave and RF Group, Centre for Applied Research in Electronics, Indian Institute of Technology Delhi, New Delhi 110016, India (e-mail: richa_b.cstaff@care.iitd.ac.in; shiban_koul@ hotmail.com).

S. Swaisaenyakorn and J. C. Batchelor are with the School of Engineering and Digital Arts, University of Kent, Canterbury CT2 7NT, U.K. (e-mail: ss638@kentforlife.net; j.c.batchelor@kent.ac.uk).

A. Alomainy is with the School of Electronic Engineering and Computer Science, Queen Mary University of London, London E1 4NS, U.K. (e-mail: a.alomainy@qmul.ac.uk).
Indoor wireless systems have to cope with severe multipath scenarios due to the presence of various objects, walls, doors, windows, furniture, and the presence of human subjects [2]. Due to their extremely large bandwidth/fine time resolution, ultrawideband (UWB) signals offer a good multipath resolution and enable high-accuracy localization [6]-[9].

The UWB time-of-arrival (TOA) measurements together with trilateration or multilateration algorithms are often used in indoor location systems to obtain high-accuracy localization [6][9]. Various commercial UWB trans-receivers and modules have been designed, which provide an accuracy in the range of 10 $15 \mathrm{~cm}$ within a range of $50 \mathrm{~m}$ [7]. Existing high-accuracy UWB systems include Ubisense, time domain [7], and Xsens [8] for human body localization integrated with the microelectromechanical system inertial sensor technology. Higher accuracy (centimeter and subcentimeter) has been reported in the literature for a short-range indoor environment localization [6]-[9].

The localization accuracy depends on various factors such as range estimation error, frequency/bandwidth used, type of environment (indoor/outdoor; highly cluttered/uncluttered), base station (BS) configuration, and the performance of the communication system. The geometric configuration of BSs used to localize the target node plays an important role in the accuracy of the localization system especially when a minimum number of BSs is used [10], [11]. Generally, four BSs are used in cuboid-shape configuration to obtain high-accuracy position estimation [7], [10]-[12]. For a short-range communication and the minimum usage of the BSs, the random placement of the BSs can lead to deterioration in position estimation accuracy. As reported briefly in [12], the dilution of precision (DOP) [13], [14] that is used to measure the appropriateness of the BS placement geometry in order to obtain high-accuracy localization can be used to evaluate the effect of random BS positioning.

In this letter, detailed DOP analysis is presented, which highlights the variation in localization error due to the presence of randomly placed BSs for a short-range body-centric communication. This work mainly focuses on a short-range applicationspecific localization analysis such as rehabilitation, physiotherapy, patient monitoring, tracking progress in performance of patient/sports persons, and in entertainment domain to capture movements of artists. The results are compared with the optimized BS configuration for which realistic body-centric localization experiments are carried out in an indoor environment [12]. Different channel links, localization systems, and range es- 


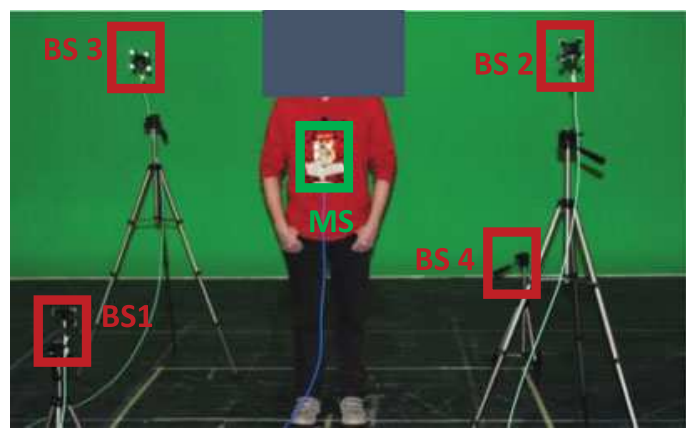

Fig. 1. Localization of wearable antenna placed on the human subject in an indoor environment with four BSs placed in an optimum position to obtain high-accuracy localization.

timation algorithms will lead to variation in range error values. The range estimation error and the standard deviation (STDEV) have been further varied for the BS configurations studied in order to obtain the trend of localization error.

\section{Methodology}

Compact tapered slot UWB antennas (TSAs) were used as wearable antennas and BSs. The measurements were performed in the UWB frequency range $(3-10 \mathrm{GHz})$ at the motion capture studio at the University of Kent, Canterbury, U.K. [12]. A human test subject $1.68 \mathrm{~m}$ tall and of average male build with a body mass index of 21.3 was chosen for localizing the wearable antennas on the body (see Fig. 1). The localization of the whole body is performed using 21 antenna positions placed on the (arms and legs) limbs and torso when the subject is standing at the center of the localization area [12].

The TSA has dimensions of $27 \mathrm{~mm} \times 16 \mathrm{~mm}$ and offers a return loss below $-10 \mathrm{~dB}$ with good radiation performance and relatively constant gain across the UWB band when off, or on a human body [15]. The four BS antennas were positioned in cuboid-shape configuration in an area of $1.8 \times 1.8 \mathrm{~m}^{2}$ in order to obtain high three-dimensional (3-D) localization accuracy [10], [12]. The frequency-domain measurements using 6400 data samples were performed using a 4-port vector network analyzer to capture $S_{21}$ parameters between each $T x-R x$ pair.

The presence of human subject leads to high-multipath and non-line-of-sight (NLOS) situations. The variation caused in the channel impulse response (CIR) due to the NLOS situations leads to positive error bias in estimation of distance between the mobile station (MS)-BS link [2], [7], [16]. This can be overcome using the NLOS identification and mitigated techniques [16], such as the algorithm in [12] that is based on the CIR characterization, TOA peak detection, and data fusion techniques [2], [16]. The algorithm in [12] is able to distinguish total lineof-sight links from the NLOS/partial NLOS with more than 95\% accuracy using the measured channel data obtained for each MS-BS link.

An accurate range estimation will lead to a high-accuracy performance of the localization system, which uses trilateration and least-square algorithm to obtain the position of the target in 3-D. Trilateration [17] consists of measuring three or more distances between the MS and BS and using these distances/range to calculate the unknown target 3-D position. The distances $r_{1}, r_{2}, r_{3}$, and $r_{4}$ are used to estimate the position of the target $\left(x_{m}, y_{m}, z_{m}\right)$ by solving the following set of equations using least square solution [17]:

$$
r_{i}^{2}=\left(x_{i}-x_{m}\right)^{2}+\left(y_{i}-y_{m}\right)^{2}+\left(z_{i}-z_{m}\right)^{2}
$$

where $i=1,2,3,4$ and $r_{i}$ represents the range measurements obtained from the distance between the MS and BS.

The localization systems require a minimum of three to four BS units to calculate 3-D positions of MSs within a room. Usually, more than three BS units are used [7], [10] to obtain high accuracy and reduce susceptibility to random measurement errors. The positioning precision depends significantly on the geometry of the BSs distribution. The geometric dilution of precision (GDOP) [13], [14] indicates the effectiveness of a geometric configuration. It is an indicator of 3-D positioning accuracy as the consequence of relative position of the BSs with respect to a MS

$$
\mathrm{GDOP}=\sqrt{\operatorname{tr}\left(H^{T} H\right)^{-1}}
$$

where

$$
H=\left[\begin{array}{llll}
a_{x 1} & a_{y 1} & a_{z 1} & 1 \\
a_{x 2} & a_{y 2} & a_{z 2} & 1 \\
a_{x 3} & a_{y 3} & a_{z 3} & 1 \\
a_{x 4} & a_{y 4} & a_{z 4} & 1
\end{array}\right]
$$

and

$$
a_{x i}=\frac{\left(x_{i}-x_{m}\right)}{r_{i}}, a_{y i}=\frac{\left(y_{i}-y_{m}\right)}{r_{i}}, a_{z i}=\frac{\left(z_{i}-z_{m}\right)}{r_{i}}
$$

where $x_{i}, y_{i}$, and $z_{i}$ correspond to the BS positions; $x_{m}, y_{m}$, and $z_{m}$ are the estimated MS positions, and $r_{i}$ is the estimated range between the mobile and each base station, and

$$
\begin{aligned}
& \mathrm{HDOP}=\sqrt{\left(\left(H^{T} H\right)^{-1}\right)_{1,1}+\left(\left(H^{T} H\right)^{-1}\right)_{2,2}} \\
& \mathrm{VDOP}=\sqrt{\left(\left(H^{T} H\right)^{-1}\right)_{3,3}}
\end{aligned}
$$

where the VDOP is the vertical dilution of precision, which is a measure of localization accuracy in 1-D [height (z-axis)], and the HDOP is the horizontal dilution of precision, which is the measure of localization accuracy in 2-D ( $x$ - and $y$-axis).

\section{RANDOM BS CONFIGURATIONS}

In order to obtain an accurate position of the target, an appropriate geometrical distribution of BSs is necessary. The optimized BS configuration chosen is a cuboid-shape configuration with four BSs each at a corner of a square with a predefined area depending upon the application. The main criteria for choosing such configuration is the achievement of high-accuracy localization results, ease of installation, cost-effectiveness, ability to replicate in any environment, symmetrical configuration, and coverage of the whole localization area of interest. The random placement of the BSs, on the other hand, can lead to degradation of the performance of the localization system, leading to inaccurate localization results of the target. In order to validate the accuracy analysis, various random BS configurations are compared with the optimized BS configuration used for the experimental data related to a body-centric localization in [9]. The range estimation errors for the wearable nodes with respect to each BS are kept the same as obtained from the experimental 
TABLE I

$X-Y$ EXAMPLE POSITIONS OF RANDOM BSS

\begin{tabular}{ccccccc}
\hline & T1 & T2 & T3 & \multicolumn{1}{c}{ T4 } & T5 & \multicolumn{1}{c}{ T6 } \\
\hline \hline BS1 & $0.2,0.3$ & $0.4,0.2$ & $0.5,0.5$ & $0.5,0$ & $0,0.8$ & $0.2,0.3$ \\
\hline BS2 & $1.5,0.5$ & $1.5,0.7$ & $1.5,0.3$ & $1.2,0.2$ & $1.8,0.4$ & $1,0.7$ \\
\hline BS3 & $0.6,1.5$ & $0.3,1.6$ & $0.3,1.5$ & $0.4,1.3$ & $0.2,1.7$ & $0.6,1.2$ \\
\hline BS4 & $1.7,1.7$ & $1.2,1.4$ & $1.3,1.3$ & $1,1.6$ & $1.6,1.2$ & $1.1,1.3$ \\
\hline
\end{tabular}
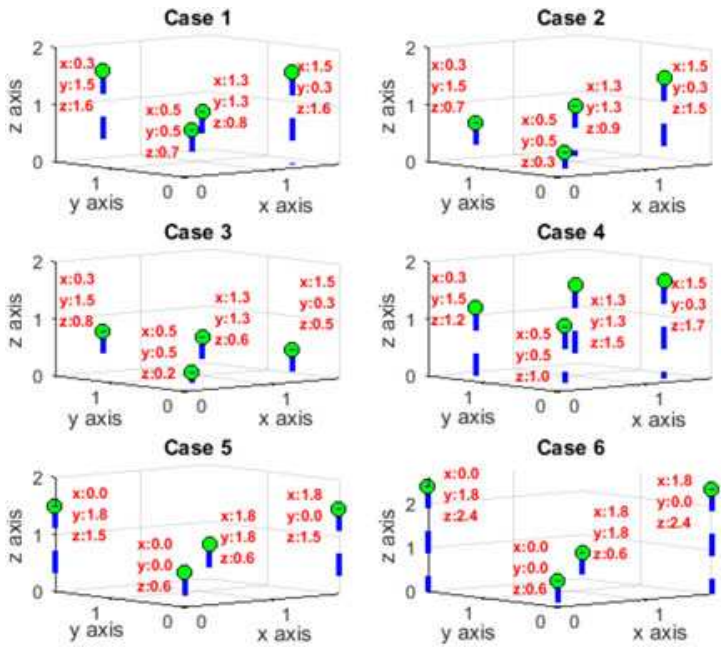

Fig. 2. Schematic of various BS configurations. Case 1-4: Random placement of BSs. Case 5: Optimized BS configuration. Case 6: Ideal BS configuration. All dimensions are in meters.

data for all the BS configurations considered so that the main focus related to the position of the BSs can be analyzed.

The six different random $x-y$ positions of the BSs are considered and for each set of $x-y$ positions, and four different random $z$-axis positions are also considered. Table I lists the random $x-y$ axis positions for the evaluation of variation in localization accuracy for body-centric applications. An example related to the BS configuration analysis is shown in Fig. 2 for the various schematics of the BS configuration. Fixed values for random $x$ - and $y$-positions for the four BSs and variable $z$ axis coordinate values for the BSs are considered for cases 1-4 (C1-C4). These results are compared with case 5 (C5) (optimized) and case 6 (C6) (ideal scenario, where distance between all BSs in $x$-, $y$-, and $z$-axis direction is the same).

\section{LOCALIZATION ACCURACY ANALYSIS}

It is observed that the trilateration and the least-square algorithm give best results when the antennas are placed at the corners of a square (C5 and C6) with 1-2 cm accuracy. Also, the height and symmetry of the $z$-axis position of the BS placement play an important role in localization accuracy. The DOP values are presented in Fig. 3 for all the random and optimal BS configurations. Average 3-D localization results are shown in Fig. 4 considering all six cases presented in Fig. 2. From the analysis, it can be concluded that localization results are directly related to the GDOP values. The $x-y$-axis localization results are more specifically related to the HDOP values, and the $z$-axis localization results are related to the VDOP values. $\mathrm{C} 1$ and $\mathrm{C} 2$ show some decrease $(0.5-1 \mathrm{~cm})$ in localization accuracy when compared with cases 5 and 6 . For such scenarios, the DOP values
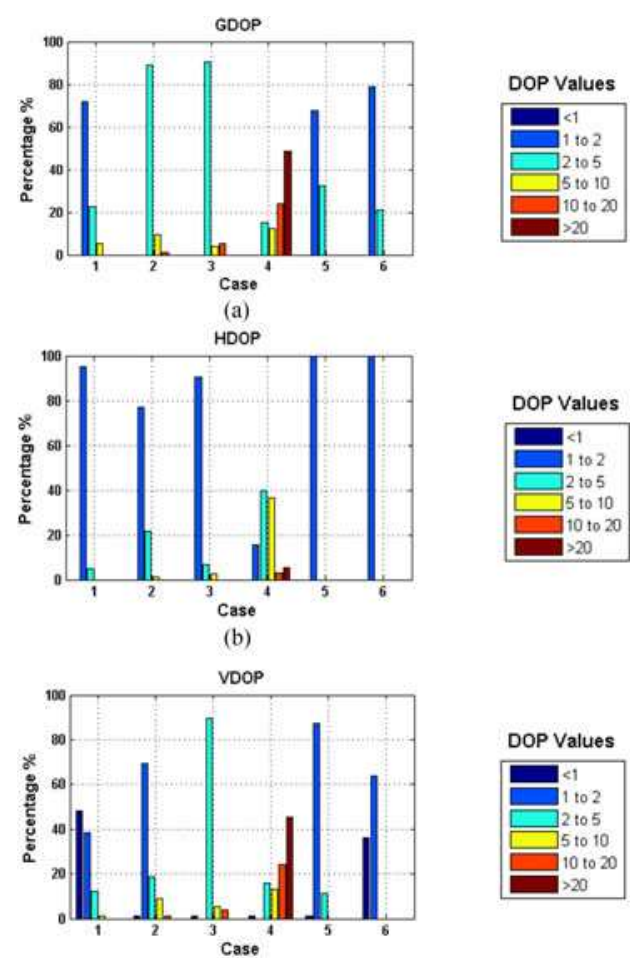

(c)

Fig. 3. DOP values (a) GDOP, (b) HDOP, and (c) VDOP, classified in different range for various BS configurations (C1-C6).

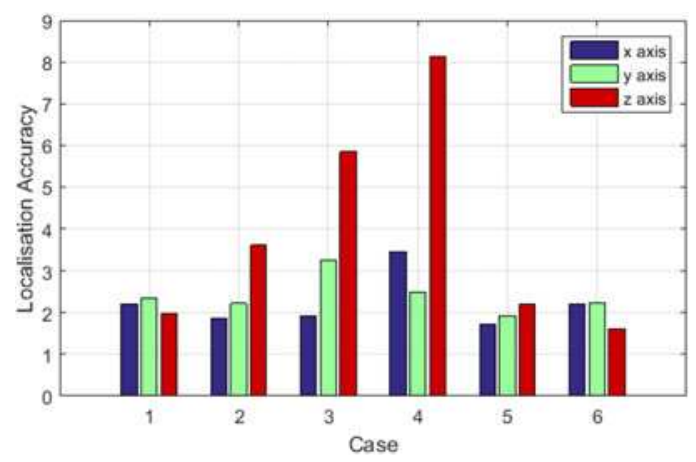

Fig. 4. Localization accuracy for various BS configurations (C1-C6).

are similar to case 5 and case 6 and fall within $0.5-5$ range. $\mathrm{C} 1$ shows lower VDOP values leading to higher accuracy for the $z$-coordinate, whereas case 2 is vice versa. C3 shows lower localization accuracy $(2-6 \mathrm{~cm})$ in comparison to the configuration used in $\mathrm{C} 1$ and $\mathrm{C} 2$ due to larger DOP values. For C3 and C4, zaxis direction values are least accurate as several VDOP values are greater than 20 . When the DOP values are $>20$, the poor localization results are obtained with an increase in localization error by 2 to 4 times when compared with optimized scenario. For C5 and C6, the localization accuracy results are quite similar as are the DOP values, which fall under high-accuracy range of $1-3$. For C6, $z$-coordinate accuracy is higher than that of $\mathrm{C} 5$ as the distance of the BSs in the $z$-direction is larger for C6, but with some deterioration in $x-y$-axis coordinate localization results.

Varying the BSs $z$-axis coordinates and keeping the $x$-y-axis coordinates optimized (C5) led to different localization results in comparison to varying all the $x y z$-coordinates. Random $z$ values chosen are shown in Fig. 2 (C1-C4), and the results are 
compared with optimized BS configuration (C5). The results indicate significant deterioration in position estimation accuracy by $2-5 \mathrm{~cm}$ for the $z$-axis coordinate for the randomly positioned $\mathrm{BSs}$ in the $z$-axis only. The $x$ - and $y$-coordinates estimation show only slight variation $(0.5-1 \mathrm{~cm})$ when compared with the optimized scenario. The $z$-axis error is high for this scenario as the nodes considered on the body are placed at different positions starting from shoulder to the ankle. Hence, more randomly positioned BSs in the $z$-axis direction will play an important role in the $z$-coordinate accuracy of the localization results.

Varying the BSs in $x-y$-axis coordinates and keeping the $z$ axis coordinates optimized has less variation in the localization results. As the human subject is standing in the center of the localization area, hence randomly placed $x-y$-axis BSs do not have very significant effect on the localization accuracy for $x$ and $y$-axis coordinates estimation. Also, as $z$-coordinate values are optimized, overall 3-D localization results are good with an average error of $2-3 \mathrm{~cm}$.

To understand the effect of varying the distance between the $\mathrm{BS}$ and MS, the BS positions have been scaled at intervals of $0.3 \mathrm{~m}$ for $x-y$-axis and $0.15 \mathrm{~m}$ for $z$-axis for a maximum area of around $5 \times 5 \mathrm{~m}^{2}$ (ten scaled positions). To perform the distance-localization error analysis, the center of the chest region is considered as a test point. Considering the optimized $\mathrm{BS}$ configuration (C5), the variation in localization accuracy is minor with an increase in distance (scaling the BS configuration) if the range estimation errors between the MS and BS pair are similar. If BSs are scaled in the xyz-direction, there can be slight increase in $(0.5 \mathrm{~cm})$ localization accuracy depending on the range error obtained. If the BSs are scaled only in the $x y$ plane, keeping $z$-coordinates the same as initial configuration, there is deterioration in the $z$-axis values by a few centimeters $(2-5 \mathrm{~cm})$, which can also be seen from the VDOP values, which increase from $(2 \rightarrow 7)$. If only $z$-axis values are scaled, there is an increase in an accuracy of the $z$-coordinate values with slight decrease in an accuracy of the $x-y$-axis values $(0.5-1 \mathrm{~cm})$. For random BS scenarios such as $\mathrm{C} 4$, having the high occurrence of the GDOP values greater than $15 / 20$, there are more chances of deterioration in localization accuracy when the configuration is scaled. This is due to more random placement of the BS configuration when the configuration is scaled over an area of $5 \times 5 \mathrm{~m}^{2}$, leading to higher GDOP values and lower accuracy of the estimated location by $2 \rightarrow 15 \mathrm{~cm}$.

The 3-D position estimation accuracy depends significantly on the error in the distance/range estimation. The distance estimation error will be different for the various types of MS-BS links formed. Hence, a theoretical analysis is carried out by varying the mean and standard deviation of a range estimation error (see Fig. 5) with values that are generally observed in practical scenarios for a short-range communication using the UWB technology, in order to study the change in position estimation error. Considering the six cases of the BS configuration as shown in Fig. 2, the mean error is varied from 1.5 to $5.5 \mathrm{~cm}$ at intervals of $1 \mathrm{~cm}$, and the standard deviation values considered are $0.6,1.2$, and $1.8 \mathrm{~cm}$. For example, the BS configuration $\mathrm{C} 4$ (random) has higher localization error (2-3 times) in comparison to the C5 (optimized) BS configuration (1.2-1.8 times) with an increase in a mean range estimation error $(1.5 \rightarrow 5.5 \mathrm{~cm})$.
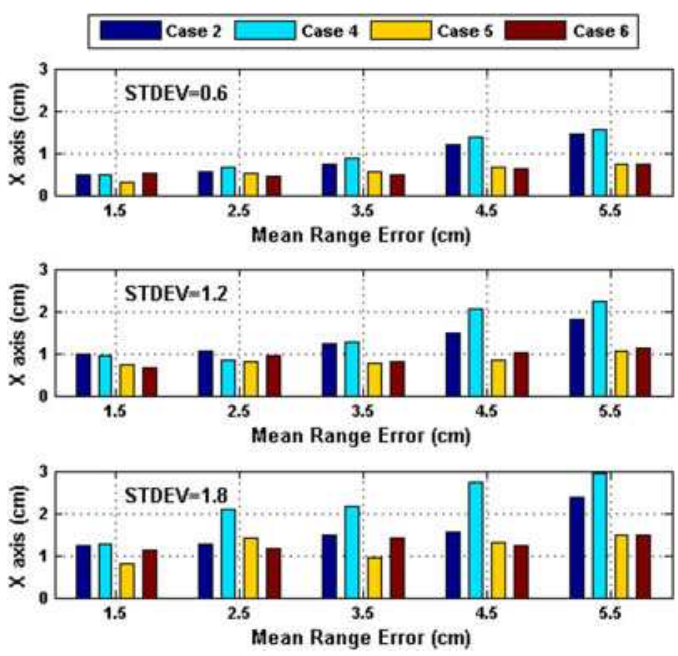

Fig. 5. $x$-axis localization accuracy for different range estimation error (STDEV: $0.6,1.2$, and $1.8 \mathrm{~cm}$ ) and variation in a mean range error from 1.5 to $5.5 \mathrm{~cm}$.

The $z$-axis magnitude of localization error is generally higher in comparison to $x-y$-axis, especially for $\mathrm{C} 4$ (three times higher), which is a random BS configuration and has the high occurrence of VDOP values larger than 20. For $z$-axis localization error, similar trend of variation in localization accuracy is observed as that of $x-y$-axis. For increase in a standard deviation, the localization error increases by $1.2-1.5$ times.

\section{CONCLUSION}

The analytical and theoretical investigations have been carried out related to the random placement of BS configurations and compared with the optimized BS configurations used for bodycentric localization. The localization accuracy analysis and the effectiveness of the BS geometrical configuration is performed using the GDOP, VDOP, and HDOP values. Higher DOP $>20$ values lead to a least accuracy with an error as high as $5-8 \mathrm{~cm}$. The DOP values in the range of 5-15 lead to decrease in localization accuracy by $1-3 \mathrm{~cm}$. Lowest DOP values (1-2) are obtained for ideal and optimized BS set up with a localization average accuracy in the range of $0.5-2 \mathrm{~cm}$. Varying only $z$-axis position of the BSs leads to higher deterioration in $z$-axis localization accuracy as the target nodes are spread out in the $z$-axis direction from the shoulder to the ankle. It is also observed that as the range estimation error for each MS-BS link increases (mean or standard deviation), there is significant increase in error for random BS configurations (two to three times) in comparison to optimized BS configurations. Future work will be to study randomly placed BS configurations in application-specific environments to analyze the variation in localization accuracy.

\section{ACKNOWLEDGMENT}

R. Bharadwaj would like to acknowledge the Science and Engineering Research Board, Department of Science and Technology, Government of India, National Postdoctoral Fellowship. 


\section{REFERENCES}

[1] H. Liu, H. Darabi, P. Banerjee, and J. Liu, "Survey of wireless indoor positioning techniques and systems," IEEE Trans. Syst., Man, Cybern. C, Appl. Rev., vol. 37, no. 6, pp. 1067-1080, Nov. 2007.

[2] S. Gezici and H. V. Poor, "Position estimation via ultra-wide-band signals" Proc. IEEE, vol. 97, no. 2, pp. 386-403, Feb. 2009.

[3] G. A. Casula, A. Michel, G. Montisci, P. Nepa, and G. Valente, "Energybased considerations for ungrounded wearable UHF antenna design," IEEE Sensors J., vol. 17, no. 3, pp. 687-694, Feb. 2017.

[4] P. Nepa and H. Rogier, "Wearable antennas for off-body radio links at VHF and UHF bands: Challenges, the state of the art, and future trends below 1 GHz," IEEE Antennas Propag. Mag., vol. 57, no. 5, pp. 30-52, Oct. 2015.

[5] X. Yang et al., "Wandering pattern sensing at S-band," IEEE J. Biomed. Health Inform., doi: 10.1109/JBHI.2017.2787595.

[6] R. Bharadwaj, S. Swaisaenyakorn, C. G. Parini, J. C. Batchelor, and A. Alomainy, "Localization of wearable ultrawideband antennas for motion capture applications," IEEE Antennas Wireless Propag. Lett., vol. 13, pp. 507-510, 2014

[7] C. Zhang, M. J. Kuhn, B. C. Merkl, A. E. Fathy, and M. R. Mahfouz, "Real-time noncoherent UWB positioning radar with millimeter range accuracy: Theory and experiment," IEEE Trans. Microw. Theory Techn., vol. 58, no. 1, pp. 9-20, Jan. 2010.

[8] Xsens Motion Grid. [Online]. Available: http://www.xsens.com/ en/general/motiongrid.

[9] C. Meier, A. Terzis, and S. Lindenmeier, "A robust 3D high precision radio location system," in Proc. Int. Symp. IEEE MTT-S Microw., 2007, pp. 397-400.
[10] R. Bharadwaj, C. G. Parini, and A. Alomainy, "Ultrawideband-based 3-D localization using compact base-station configurations," IEEE Antennas Wireless Propag. Lett., vol. 13, pp. 221-224, 2014.

[11] H. Kiwan, A. Bais, and Y. L. Morgan, "A new base stations placement approach for enhanced vehicle position estimation in parking lot," in Proc. 15th Int. Conf. IEEE Intell. Transp. Syst., Anchorage, AK, USA, 2012, pp. 1288-1293.

[12] R. Bharadwaj, S. Swaisaenyakorn, C. G. Parini, J. C. Batchelor, and A. Alomainy, "Impulse radio-ultra wideband communications for localization and tracking of human body and limbs movement for healthcare applications," IEEE Trans. Antennas Propag., vol. 65, no. 12, pp. 7298 7309, Dec. 2017.

[13] B. Li, A. G. Dempster, and J. Wang, "3D DOPs for positioning applications using range measurements," Wireless Sens. Netw., vol. 3, no. 10, pp. 334 $340,2011$.

[14] R. B. Langley, "Dilution of precision," GPS World, vol. 10, no. 5, pp. 52 59, May 1999

[15] A. Alomainy, A. Sani, A. Rahman, J. G. Santas, and Y. Hao, "Transient characteristics of wearable antennas and radio propagation channels for ultrawideband body-centric wireless communications," IEEE Trans. Antennas Propag., vol. 57, no. 4, pp. 875-884, Apr. 2009.

[16] I. Guvenc and C. Chia-Chin, "A survey on TOA based wireless localization and NLOS mitigation techniques," IEEE Commun. Surveys Tuts., vol. 11 no. 3, pp. 107-124, Jul./Sep. 2009.

[17] K. W. Cheung, H. C. So, W.-K Ma, and Y. T. Chan, "Least squares algorithms for time-of-arrival-based mobile location," IEEE Trans. Signal Process., vol. 52, no. 4, pp. 1121-1130, Apr. 2004. 